\title{
Response to: Improving postpartum screening rates
}

\author{
Hector Mendez-Figueroa $\cdot$ Donald R. Coustan
}

Published online: 1 April 2014

(C) Springer-Verlag Berlin Heidelberg 2014

We would like to thank Drs. Gupta and Kalra [1] for their comments regarding our study [2] evaluating a strategy aimed at improving postpartum glucose tolerance testing. This difficult clinical dilemma may only be overcome if the problem is addressed from different angles or in a multifactorial approach. We agree that other strategies should be evaluated in order to overcome suboptimal postpartum testing rates currently seen. These strategies should not only lead to increased detection of diabetes and prediabetes but also be cost-effective. Direct home visits, screening with hemoglobin A1C levels and self-administered OGTT may lead to increased testing rates and may be part of the solution of this complex problem. We would encourage trials of these and other methods to determine their efficacy.

Conflict of interest The authors report no conflict of interest.

\section{References}

1. Gupta Y, Karla S (2014) Improving postpartum screening rates. Arch Gynecol Obstet. doi:10.1007/s00404-014-3213-9

2. Mendez-Figueroa H, Daley J, Breault P et al (2014) Impact of an intensive follow-up program on the postpartum glucose tolerance testing rate. Arch Gynecol Obstet. doi:10.1007/s00404-014-3157-0

H. Mendez-Figueroa $(\bowtie)$

Department of Obstetrics, Gynecology and Reproductive

Sciences, The University of Texas Health Science Center at Houston, 6431 Fannin MSB 3.286, Houston, TX 77030, USA

e-mail: Hector.R.Mendezfigueroa@uth.tmc.edu

\section{R. Coustan}

Division of Maternal-Fetal Medicine, Department of Obstetrics and Gynecology, Women and Infants' Hospital, Warren Alpert Medical School of Brown University, Providence, USA 